\title{
Questes
}

vestes Revue pluridisciplinaire d'études médiévales

11 | 2007

La transmission

\section{La transmission : éléments bibliographiques}

Sophie Albert et Valérie Deplaigne

\section{(2) OpenEdition}

\section{Journals}

Édition électronique

URL : http://journals.openedition.org/questes/644

DOI : 10.4000/questes.644

ISSN : 2109-9472

\section{Éditeur}

Les Amis de Questes

Édition imprimée

Date de publication : 15 mars 2007

Pagination : 96-105

ISSN : 2102-7188

\section{Référence électronique}

Sophie Albert et Valérie Deplaigne, «La transmission : éléments bibliographiques », Questes [En ligne], 11 | 2007, mis en ligne le 01 janvier 2014, consulté le 15 septembre 2020. URL : http:// journals.openedition.org/questes/644 


\title{
BIBLIOGRAPHIE
}

\author{
Sophie ALBERT, \\ avec l'aide de Valérie DEPLAIGNE
}

\section{La transmission des textes}

- ANTIQUITÉ ET MOYEN AGE

ADHEMAR, Jean, Influences antiques dans l'art $d u$ Moyen Âge français. Recherches sur les sources et les thèmes d'inspiration, Paris, Éditions du CTHS, 1996 ( $1^{\text {ère }}$ éd. 1939).

BADAWI, Abdurrahman, La Transmission de la philosophie grecque au monde arabe, Paris, Vrin, 1968.

BERTHELOT, Anne, BRUSEGAN, Rosanna, BUSCHINGER, Danielle, et ZIRONI, Alessandro (éd.), L'Antichità nella cultura europea del Medioevo. Ergebnisse der internationalen Tagung in Padua (27/09-01/10/1997), Greifswald, Reineke-Verlag, 1998.

BRAET, Herman, VERBEKE, Werner, et WELKENHUYSEN, Andries (éd.), Mediaeval Antiquity, Louvain, Leuven University Press, 1995.

CAVALLO, Guglielmo, Dalla parte del libro. Storie di trasmissione dei classici, Urbino, QuattroVenti, 2002.

CHAVANNES-MAZEL, Claudine A., et SMITH, Margaret M. (éd.), Medieval Manuscripts of the Latin Classics: Production and Use. Proceedings of the Seminar in the History of the Book to 1500, Leiden, 1993, Los Altos Hills (California), Anderson-Lovelace / London, The Red Gull Press, 1996.

COURCELLE, Pierre, Les Lettres grecques en Occident de Macrobe à Cassiodore, Paris, De Boccard, 1953.

CURTIUS, Ernst Robert, La Littérature européenne et le Moyen Âge latin, trad. française Jean Bréjoux, Paris, Presses Universitaires de France, 1986 (1 ère éd. 1956). 
HAMMAN, Adalbert-Gautier, L'Épopée du livre: la transmission des textes anciens, du scribe à l'imprimerie, Paris, Perrin, 1985.

MONFRIN, Jacques, «La connaissance de l'Antiquité et le problème de l'Humanisme en langue vulgaire dans la France du $\mathrm{XV}^{\mathrm{e}}$ siècle ", The Late Middle Ages and the Dawn of Humanism Outside Italy, Louvain, Leuven University Press / The Hague, Martinus Nijhoff, 1972, p. 131-170.

MUNK OLSEN, Birger, L'Étude des auteurs classiques latins aux XI et XII siècles, Paris, Éditions du CNRS, 1982-1989.

MUNK OLSEN, Birger, "La popularité des textes classiques entre le $\mathrm{IX}^{\mathrm{e}}$ et XII ${ }^{\mathrm{e}}$ siècles ", Revue d'histoire des textes, 14-15 (1984-1985), p. 169-181.

PANOFSKY, Erwin, et SAXL, Fritz, La Mythologie classique dans l'art médiéval, trad. de Sylvie Girard (traduit de Classical Mythology in Mediaeval Art, article paru en 1933), Saint-Pierre-de-Salerne, Gérard Monfort, 1990.

La Réception de la littérature classique au Moyen Âge (IX ${ }^{e}-X I I^{e}$ siècle). Choix d'articles publié par des collègues à l'occasion de son soixantième anniversaire, pour Birger Munk Olsen, Copenhagen, Museum Tusculanum Press, Université de Copenhague, 1995.

RENUCCI, Paul, L'Aventure de l'humanisme européen au Moyen Âge (IV ${ }^{e}$ $X I V^{e}$ siècles), Paris, Les Belles Lettres, 1953.

REYNOLDS, Leighton Durham, et WILSON, Nigel Guy, D'Homère à Érasme: la transmission des classiques grecs et latins, trad. par Claude Bertrand et mis à jour par Pierre Petitmengin (traduit de Scribes and Scholars: A Guide to the Transmission of Greek and Latin Literature, Oxford, Clarendon Press, 1991), Paris, Éditions du CNRS, 1988 (nouvelle éd. revue et augmentée).

SEZNEC, Jean, La Survivance des dieux antiques, Paris, Champion, 1993 (1 ${ }^{\text {ère }}$ éd. 1939).

TREMBLAY, Florent A., Les Classiques au Moyen Âge: l'éducation au Moyen Age, Lewiston / New York, Edwin Mellen Press, 1988.

VALVO, Alfredo, La Diffusione dell'eredità classica nell'età tardoantica e medievale: forme e modi di transmissione. Atti del seminario nazionale (Trieste, 19-20 settembre 1996), Alessandria, Edizioni dell'Orso, 1997. 


\section{- TRANSMISSION DES TEXTES AU SEIN DU MOYEN AGE}

ALVERNY (d'), Marie-Thérèse, La Transmission des textes philosophiques et scientifiques au Moyen Âge, recueil d'articles éd.par Charles Burnett, Aldershot, Variorum, 1994.

BANNIARD, Michel, Viva Voce. Communication écrite et communication orale $d u I V^{e}$ au $I X^{e}$ siècle en Occident latin, Paris, Institut des études augustiniennes, 1992.

BISCHOFF, Bernhard, Manuscripts and Libraries in the Age of Charlemagne, trad. Michael Gorman (traduit de Mittelalterliche Studien: ausgewählte Aufsätze zur Schriftkunde und Literaturgeschichte I-III, à l'exception du chapitre 7), Cambridge, Cambridge University Press, 1995.

BOENIG, Robert, et DAVIS, Kathleen, Manuscript, Narrative, Lexicon: Essays on Literary and Cultural Transmission in Honor of Whitney F. Bolton, Lewisburg, Bucknell University Press / London, Associated University Presses, 2000 .

BOITANI, Piero, MANCINI, Mario, et VÀRVARO, Alberto (éd.), Lo Spazio letterario del Medioevo. II : Il medioevo volgare, vol. 2, La circolazione del testo, Roma, Salerno, 2002.

CASTALDI, Lucia, et CHIESA, Paolo, La Trasmissione dei testi latini del Medioevo. Mediaeval latin texts and their transmission (TE.TRA. I et II), Florence, SISMEL, Edizioni del Galluzzo, 2 vol., 2004 et 2005.

CLANCHY, Michael T., From Memory to Written Record: England, 10661307, London, E. Arnold, 1979 (2 éd. Oxford / Cambridge, Blackwell, 1993).

CRICK, Julia, et WALSHAM, Alexandra, The Uses of Script and Print, 13001700, Cambridge / New York, Cambridge University Press, 2004.

DOANE, Alger Nicolaus, et PASTERNACK, Carol Braun, Vox intexta. Orality and Textuality in the Middle Ages (Conference Con-Texts at Madison, Wisconsin, in April 1988), Madison, University of Wisconsin Press, 1991.

FATTORI, Marta, et HAMESSE, Jacqueline (éd.), Rencontres de cultures dans la philosophie médiévale: traductions et traducteurs de l'Antiquité tardive au XIV ${ }^{e}$ siècle. Actes du Colloque international de Cassino, 15-17 juin 1989, organisé par la Société internationale pour l'étude de la philosophie médiévale 
et l'Università degli studi di Cassino, Louvain-la-Neuve, Université catholique de Louvain / Cassino, Università degli studi di Cassino, 1990.

KELLY, Douglas Floyd (éd.), The Medieval Opus: Imitation, Rewriting and Transmission in the French Tradition. Proceedings of the Symposium held at the Institute for Research in Humanities (October 5-7 1995, the University of Wisconsin-Madison), Amsterdam, Rodopi, 1996.

MINNIS, Alastair J. (éd.), Late-medieval Religious Texts and their Transmission: Essays in honour of A. I. Doyle. Proceedings of the sixth York Manuscripts Conference, Woodbridge / Rochester, D. S. Brewer, 1994.

PARKES, Malcolm Beckwith, Scribes, Scripts and Readers: Studies in the Communication, Presentation and Dissemination of Medieval Texts, London / Rio Grande (Ohio), The Hambledon Press, 1991.

TYSSENS, Madeleine, "Aspects de la transmission des textes littéraires au Moyen Âge ", Bulletin de la Classe des Lettres et des Sciences morales et politiques. Académie Royale de Belgique, n 7-12 (1996), p. 515-540.

- MONOGRAPHIES

BADEL, Pierre-Yves, Le Roman de la Rose au XIV siècle. Études de la réception de l'œuvre, Genève, Droz, 1980.

COURCELLE, Pierre, La Consolation de Philosophie dans la tradition littéraire, antécédents et postérité de Boèce, Paris, Études augustiniennes, 1967.

COURCELLE, Pierre, Lecteurs païens et lecteurs chrétiens de l'Énéide, 2 vol. (1. Les Témoignages littéraires; 2. Les Manuscrits illustrés de l'Énéide $d u X^{e}$ au $X V^{e}$ siècle, avec la collab. de Jeanne Courcelle), Paris, Gauthier-Villars, diff. De Boccard, 2 vol., 1984.

CRICK, Julia, The Historia Regum Britannie of Geoffrey of Monmouth. IV : Dissemination and Reception in the later Middle Ages, Cambridge, D. S. Brewer, 1991.

HUOT, Sylvia Jean, The Romance of the rose and its Medieval Readers: interpretation, reception, manuscript transmission, Cambridge / New York / Victoria (Australia), Cambridge University Press, 1993.

KASTER, Robert Andrew, The Tradition of the Text of the Aeneid in the Ninth Century, New York, Garland Publications, 1990. 
Lectures médiévales de Virgile, Rome, École française de Rome, 1985.

MAGGIONI, Giovanni Paolo, Ricerche sulla composizione e sulla trasmissione della Legenda aurea, Spoleto, Centro Italiano di Studi sull'Alto Medioevo, 1995.

MORA-LEBRUN, Francine, L'Énéide médiévale et la naissance du roman, Paris, Presses Universitaires de France, 1994.

TURCAN-VERKERK, Anne-Marie, Les Manuscrits de la Charité, Cheminon et Montier-en-Argonne: collections cisterciennes et voies de transmission des textes (IX $-X I X^{e}$ siècles), Paris, Éditions du CNRS, 2000.

\section{Transmission du savoir et des techniques}

BRAUNSTEIN, Philippe, "Savoir et savoir-faire : les transferts techniques », L'Innovation technique au Moyen Âge. Actes du VI Congrès international d'archéologie médiévale, 1-5 octobre 1996, Dijon, Mont-Beuvray, Chenôve, Le Creusot, Montbard, éd. Patrice Beck, Paris, Errance, 1998, p. 303-309.

BRAUNSTEIN, Philippe, «Maîtrise et transmission des connaissances techniques au Moyen Âge », History of Technology 21 (1999), p. 155-165.

COMET, Georges (dir), La Transmission des connaissances techniques, Aixen-Provence, Publications de l'Université, 1995.

DELUMEAU, Jean (dir.), La Religion de ma mère. Le rôle des femmes dans la transmission de la foi, Paris, Cerf, 1992.

Les Entrées dans la vie: initiations et apprentissages. XII Congrès de la SHMESP (Nancy, 1981), Nancy, Presses Universitaires de Nancy, 1982.

GLICK, Thomas F., Irrigation and Hydraulic Technology: Medieval Spain and its Legacy, Aldershot, Variorum, 1996.

GRECI, Roberto, Corporazioni e mondo del lavoro nell'Italia padana medievale, Bologne, CLUEB, 1988.

LUSIGNAN, Serge, et de RIDDER-SYMOENS, Hilde (dir.), Transfert des savoirs et créations d'universités. The Transmission of Learning and the Creation of Universities, Paedagogica Historica, numéro spécial, 34/2 (1998). 
MICHAUD, Francine, "Apprentissage et salariat à Marseille avant la Peste Noire », Revue historique 278 (1994), p. 3-36.

NOBEL, Pierre (dir.), La Transmission des savoirs au Moyen Âge et à la Renaissance. Volume 1, du XII au XV $V^{e}$ siècle, Besançon, Presses Universitaires de Franche-Comté, 2005.

RICHÉ, Pierre, Éducation et culture dans l'Occident barbare, Paris, Seuil, 1962.

RICHÉ, Pierre, et VERGER, Jacques, Des Nains sur des épaules de géants : maîtres et élèves au Moyen Âge, Paris, Tallandier, 2006.

SIGAL, Pierre-André (éd.), Éducation, apprentissages, initiation au Moyen Âge. Actes du premier colloque international de Montpellier, Université PaulValery, novembre 1991, Montpellier, CRISIMA, 1993 (Cahiers du CRISIMA, $\left.\mathrm{n}^{\circ} 1\right)$.

VERGER, Jacques (dir.), Éducations médiévales: l'enfance, l'école, l'Église en Occident $V^{e}-X V^{e}$ siècles), Paris, Service d'histoire de l'éducation, INRP, 1991.

VERGER, Jacques, Culture, enseignement et société en Occident aux XII et XIII siècles, Rennes, Presses Universitaires de Rennes, 1999.

\section{Enjeux lignagers, sociaux et politiques de la transmission}

BARTHELEMY, Dominique, "Parenté », Histoire de la vie privée. II. De l'Europe féodale à la Renaissance, dir. Philippe ARIES et Georges DUBY, Paris, Seuil, 1985, p. 96-161.

BEAUCAMP, Joëlle, et DAGRON, Gilbert, La Transmission du patrimoine : Byzance et l'aire méditerranéenne, Paris, De Boccard, 1998.

BOUCHARD, Constance Brittain, Those of my Blood: Constructing Noble Families in Medieval Francia, Philadelphia, University of Pennsylvania Press, 2001.

BOUGARD, François, FELLER, Laurent et LE JAN, Régine (éd.), Dots et douaires dans le haut Moyen Âge. Actes de la Table Ronde de Lille IIIValenciennes (mars 2000), Rome, École française de Rome, 2002. 
BOUGARD, François, LA ROCCA, Cristina et LE JAN, Régine (éd.), Sauver son âme et se perpétuer : transmission du patrimoine et mémoire au haut Moyen Âge. Actes de la table ronde "Salvarsi l'anima, perpetuare la famiglia » réunie à Padoue les 3, 4 et 5 octobre 2002, Rome/Paris, École française de Rome/diffusion De Boccard, 2005.

CARBALLEIRA DEBASA, Ana María, Legados pios y fundaciones familiares en Al-Andalus : siglos IV/X-VI/XII, Madrid, Consejo superior de investigaciones científicas, 2002.

CARRON, Roland, Enfant et parenté dans la France médiévale ( $X^{e}-$ XIII ${ }^{e}$ siècles), Genève/Paris, Droz/diffusion Champion-Slatkine, 1989.

COLLAS, Alain, «Le père, l'héritier et l'ancêtre. Quelques images de la parenté chez les notables urbains au $\mathrm{XV}^{\mathrm{e}}$ siècle : l'exemple de Bourges », Revue historique 589 (janvier-mars 1994), p. 37-50.

CORBIER, Mireille (éd.), Adoption et fosterage, Paris, De Boccard, 1999.

COURTEMANCHE, Andrée, La Richesse des femmes : patrimoine et gestion à Manosque au XIV siècle, Montréal/Paris, Bellarmin/Vrin, 1993.

COURTEMANCHE, Danielle, Euvrer pour la postérité. Les testaments parisiens des gens du roi au début du XV ${ }^{e}$ siècle, Paris, L'Harmattan, 1997.

COURTEMANCHE, Danielle, "Le testament parisien au tournant du $\mathrm{XV}^{\mathrm{e}}$ siècle : une courroie de transmission d'acquisition du savoir », Moyen Âge 105/1 (1999), p. 55-70.

DELUMEAU, Jean, et ROCHE, Daniel (dir.), Histoire des pères et de la paternité, Paris, librairie Larousse, 1990.

DESCLAIS-BERKVAM, Doris, Enfance et maternité dans la littérature française des XII ${ }^{e}$ et XIII ${ }^{e}$ siècles, Paris, Champion, 1981.

DUBY, Georges, La Société chevaleresque. Hommes et structures du Moyen Âge. Paris, Flammarion, 1988 (1 $1^{\text {ère }}$ éd. Paris, éditions de l'École des Hautes Études en Sciences Sociales, 1979).

DUBY, Georges, et LE GOFF, Jacques (éd.), Famille et parenté dans l'Occident médiéval. Actes du colloque de Paris (6-8 juin 1974) organisé par l'École Pratique des Hautes Études (VI section), Rome, École française de Rome, 1977. 
GASPARRI, Stefano, et LA ROCCA, Cristina, Carte di famiglia. Strategie, rappresentazione e memoria del gruppo familiare di Totone di Campione (721877), Rome, Viella, 2005.

GEARY, Patrick J., Furta Sacra: Thefts of Relics in the Central Middle Ages, Princeton, Princeton University Press, 1978 (trad. française : Le Vol des reliques au Moyen Age: furta sacra, trad. de l'anglais par Pierre-Emmanuel Dauzat, Paris, Aubier, 1993).

GEARY, Patrick J, Phantoms of Remembrance: Memory and Oblivion at the End of the First Millennium, Princeton, Princeton University Press, 1994 (trad. française : Mémoire et oubli à la fin du premier millénaire, trad. de l'anglais par Jean-Pierre Ricard, Paris, Aubier, diffusion Flammarion, 1996).

GÉNICOT, Léopold, Les Généalogies, coll. «Typologie des sources du Moyen Âge occidental », t. XV, Brepols, Turnhout, 1975.

GOEZ, Werner, Translatio imperii. Ein Beitrag zur Geschichte des Geschichtsdenkens und der politischen Theorien im Mittelalter und in der frühen Neuzeit, Tübingen, Mohr, 1958.

GOODY, Jack, The Development of the Family and Marriage in Europe, Cambridge / New York, Cambridge University Press, 1983 (trad. française : L'Évolution de la famille et du mariage en Europe, Paris, Armand Colin, 1985).

GUENÉE, Bernard, «La culture historique des nobles : le succès des Faits des Romains (XIII $-\mathrm{XV}^{\mathrm{e}}$ siècle) », La Noblesse au Moyen Âge (XI $-X V^{e}$ siècle). Essais à la mémoire de Robert Boutruche, éd. Philippe Contamine, Paris, Presses Universitaires de France, 1976, p. 261-288.

GUERREAU-JALABERT, Anita, «Sur les structures de parenté dans l’Europe médiévale », Annales ESC 36 (nov.-déc. 1981), p. 1028-1049.

HAUGEARD, Philippe, $D u$ Roman de Thèbes à Renaut de Montauban : une genèse sociale des représentations familiales, Paris, Presses Universitaires de France, 2002.

JACOB, Robert, Les Époux, le seigneur et la cité: coutume et pratiques matrimoniales des bourgeois et paysans de France du Nord au Moyen Âge, Bruxelles, Facultés universitaires Saint-Louis, 1990. 
JUSSEN, Bernhard, Patenschaft und Adoption im frühen Mittelalter : künstliche Verwandtschaft als soziale Praxis, Göttingen, Vandenhoeck und Ruprecht, 1991 (trad. anglaise par Pamela Eve Selwyn, Spiritual Kinship as Social Practice: Godparenthood and Adoption in the Early Middle Ages, 2000).

KLAPISCH-ZUBER, Christiane, La Maison et le nom. Stratégies et rituels dans L'Italie de la Renaissance, Paris, Éditions de l'EHESS, 1990.

KLAPISCH-ZUBER, Christiane, L'Ombre des ancêtres. Essai sur l'imaginaire médiéval de la parenté, Paris, Fayard, 2000.

LABORDERIE, Olivier de, "Les généalogies des rois d'Angleterre sur rouleaux manuscrits (milieu XIII ${ }^{\mathrm{e}}$ siècle-début $\mathrm{XV}^{\mathrm{e}}$ siècle) : conception, diffusion et fonctions », La Généalogie entre science et passion (120 Congrès national des sociétés historiques et scientifiques, section ethnologie et anthropologie françaises, Aix-en-Provence, 1995, éd. Tiphaine Barthélémy et Marie-Claude Pingaud, Paris, Éditions du CTHS, 1997, p. 181-199.

LE JAN, Régine, Famille et pouvoir dans le monde franc (VII $-X^{e}$ siècle). Essai d'anthropologie sociale, Paris, Publications de la Sorbonne, 1995.

LETT, Didier, "'L'expression du visage paternel'. La ressemblance entre le père et le fils à la fin du Moyen Âge : un mode d'appropriation symbolique », Cahiers de Recherches Médiévales (XIII ${ }^{e}-X V^{e}$ siècles), Etre père à la fin du Moyen Âge, nº 4 (1997), p. 115-125.

LEWIS, Andrew W., Royal Succession in Capetian France: Studies on Familial Order and the State, Cambridge (Mass.), Harvard University Press, 1981 (trad. française : Le Sang royal : la famille capétienne et l'État, France, $X^{e}-$ $X I V^{e}$ siècle, trad. de l'anglais par Jeannie Carlier, Paris, Gallimard, 1986).

Liens de famille: vivre et choisir sa parenté. Médiévales 19, numéro spécial, Presses Universitaires de Vincennes, automne 1990.

LORCIN, Marie-Thérèse, Vivre et mourir en Lyonnais à la fin du Moyen Âge, Paris, Éditions du CNRS, 1981.

MAUREL, Christian, "Structures familiales et solidarités lignagères à Marseille au $\mathrm{XV}^{\mathrm{e}}$ siècle : autour de l'ascension sociale des Forbin », Annales ESC 41 (mai-juin 1986), p. 657-681.

MAURICE, Philippe, La Famille en Gévaudan au $X V^{e}$ siècle, Paris, Publications de la Sorbonne, 1998. 
MUSSET, Jacqueline, «Les gains de survie de la veuve et du veuf normands au $\mathrm{XV}^{\mathrm{e}}$ siècle : douaire et droit de viduité ", Colloque sur Marguerite de Lorraine et son temps, Bulletin de la Société historique et archéologique de l'Orne, 1989, p. 123-132.

NASSIET, Michel, « Nom et blason », L'Homme 129 (1994), p. 5-30.

NASSIET, Michel, Parenté, noblesse et états dynastiques : $X V^{e}-X V I^{e}$ siècles, Paris, Editions de l'EHESS, 2000.

POUMARÈDE, Jacques, Les Successions dans le sud-ouest de la France au Moyen Âge: géographie coutumière et mutations sociales, préface de Paul Ourliac, Paris, Presses Universitaires de France, 1972.

Les Relations de parenté dans le monde médiéval. Actes du $14^{e}$ colloque du Centre universitaire d'études et de recherches médiévales d'Aix, Aix-enProvence, CUERMA, 1989 (Senefiance, 26).

RÖCKELEIN, Hedwig, Reliquientranslationen nach Sachsen im 9. Jahrhundert: über Kommunikation, Mobilität und Öffentlichkeit im Frühmittelalter, Stuttgart, J. Thorbecke, 2002.

SIZARET, Louis, Essai sur l'histoire de la dévolution successorale ab intestat $d u V^{e}$ au $X^{e}$ siècle dans les pays de l'ancienne Gaule, Dijon, Centre de Recherches Historiques, Faculté de droit et de science politique, 1975.

SMAIL, Daniel Lord, « Démanteler le patrimoine. Les femmes et les biens dans la Marseille médiévale », Annales : histoire et sciences sociales 52/2 (1997), p. 343-368.

Les Transferts patrimoniaux en Europe occidentale (VII ${ }^{e} X^{e}$ siècles), Mélanges de l'École française de Rome (MEFRM) 111/2, 1999.

WHITE, Stephen D., Custom, Kinship and Gifts to Saints: the "Laudatio parentum " in Western France, 1050-1150, Chapel Hill, University of North Carolina press, 1988.

YVER, Jean, Égalité entre héritiers et exclusion des enfants dotés : essai de géographie coutumière, Paris, Sirey, 1966. 